\title{
COMMUNICATION AND CONTROL IN SMALL BATCH PART MANUFACTURING
}

\author{
J. J. Tiemersma, ${ }^{*}$ W. CurTis $\dagger$ and H. J. J. Kals* \\ * Laboratory of Production Engineering, University of Twente, The Netherlands and $†$ ICL, Manchester, U.K.
}

\begin{abstract}
This paper reports on the development of a real-time control network as an integrated part of a shop floor control system for small batch part manufacturing. The shop floor control system is called the production control system (PCS). The PCS aims at an improved control of small batch part manufacturing systems, enabling both a more flexible use of resources and a decrease in the economical batch size. For this, the PCS integrates various control functions such as scheduling, dispatching, workstation control and monitoring, whilst being connected on-line to the production equipment on the shop floor. The PCS can be applied irrespective of the level of automation on the shop floor. The control network is an essential part of the PCS, as it provides a real-time connection between the different modules (computers) of the PCS, which are geographically distributed over the shop floor. An overview of the requirements of such a control network is given. The description of the design includes the services developed, the protocols used and the physical layout of the network. A prototype of the PCS, including the control network, has been installed and tested in a pilot plant. The control network has proven that it can supply a manufacturing environment, consisting of equipment from different vendors with different levels of automation, with a reliable, low cost, real-time communication facility.
\end{abstract}

\section{INTRODUCTION}

From the beginning of the eighties, research in manufacturing has focused on the development of computer-integrated manufacturing (CIM) systems. CIM should provide industry with opportunities to streamline production flows, to reduce lead times, to decrease stock volumes and to increase overall quality while adapting the enterprise fully to the needs of the market. The design of a CIM structure is based on a "top-down" approach, where the overall activities can be analysed and broken down into a set of consistent but semi-independent functions. One of the functions of a CIM system is shop floor control (SFC) which addresses a very complex area. This particularly applies to small batch part manufacturing, because of the highly non-repetitive nature of the manufacturing activities and the corresponding diversity of the information flows. SFC closes the loop between the planning and the execution phases of the manufacturing process. It is responsible for the overall control of the

Acknowledgement-The authors express their thanks to the members of the ESPRIT 809 project, Dextralog Ltd, Blackburn, U.K., ICL, Manchester, U.K., Krupp Atlas Datensysteme, Essen, Germany, Delft University of Technology, Delft, The Netherlands, Laboratory of Production Engineering of the University of Twente, Enschede, The Netherlands and Morskate Aandrijvingen B.V., Hengelo, The Netherlands. flow of production orders and of the attendant information flows on the shop floor. The major deficiencies in part manufacturing nowadays are both the lack of integration of the various planning, control and monitoring functions and the lack of on-line communication between these functions and the equipment on the shop floor.

This paper deals with the development of a realtime control network of a SFC system, providing full integration of the control and monitoring functions and the manufacturing equipment on the shop floor.

The paper has been divided into five sections. Section 1 gives a brief overview of the development of the PCS. A description is given of the manufacturing environment controlled by the PCS, the requirements of a shop floor control system and the functions and architecture of the PCS. Section 2 describes the (design) requirements of the control network following from the different functions of the PCS and the international communication standards for networks. Section 3 gives an overview of the design of the control network. Finally, in Sections 4 and 5, the results and conclusions are presented including aspects of performance and reliability.

\section{PRODUCTION CONTROL SYSTEM (PCS)}

This section gives an introduction to the development of the PCS. ${ }^{6}$ First the target manufacturing environment is described and a definition is given of shop 
floor control. Then the functions and architecture of the PCS are briefly described.

\subsection{Small batch part manufacturing}

Small batch part manufacturing can be characterized by an average batch size of 50 , but ranges from 1 to 1000. General purpose machines are used to produce mostly new products, with a large variety and short lead times. A functional description of the manufacturing environment controlled by the PCS is given by using the CIM reference model shown in Fig. 1. The various manufacturing functions involved are distributed over five hierarchical levels.

The functions at factory level control the overall manufacturing system and are used to communicate with the customers and other external sources. At this level the orders enter the factory via the sales department. The technical product data is generated or imported and evaluated by the design and engineering departments.

At system level process planning, master planning, material planning, capacity planning and quality assurance take place. At this level how a product will be manufactured is determined (which operations, sequences, equipment used, tools used, etc.). Master schedules are set up, based on the capacity of the manufacturing equipment, the estimated manufacturing times and the due dates of the orders. Capacity plans are the output from the system level.

From the next lower level, the cell level, to the equipment level all activities are directed towards the execution of the capacity plans. A manufacturing system may contain more cells, each having its own system for the control of the material and information flow. Important cell functions are scheduling, dispatching, monitoring, diagnostics and transport of tools, materials, part programs, presetting data, instructions for quality control, etc.
The second lowest level is the workstation level, where the transformation of workpieces takes place. A workstation is addressed via its control unit which in its turn addresses one or more (e.g. concurrent working) pieces of computer controlled equipment and/or operators. The latter indicates that a workstation controller may also address an operator by sending him control and manual task instructions. A few examples of workstations are given in the CIM reference model.

The lowest level is called the equipment level where machining and manual operations are executed.

The PCS deals with shop floor control and is located at cell level, workstation level and equipment level in the CIM reference model. SFC consists of those activities that are directed towards the transformation of orders planned at the system level into completed products, in an efficient and cost-effective manner. It governs the very short-term detailed planning (scheduling), dispatching and monitoring activities needed at the shop floor.

The design of the PCS has been aimed at manufacturing cells equipped with automated workstations coming from different vendors. In the case of a low degree of automation the PCS can send information and instructions to human operators via terminals. So the PCS allows a gradual implementation of automated control whereby terminals and operators may gradually be exchanged for automated equipment whenever this becomes available.

\subsection{Requirements for a shop floor control system}

The requirements for a shop floor control system are that it should be effective, flexible, adaptable, humanoriented and low-cost. These requirements are briefly described below.

Effectiveness is defined as the capacity of the PCS to execute all the plans released to the shop floor in a

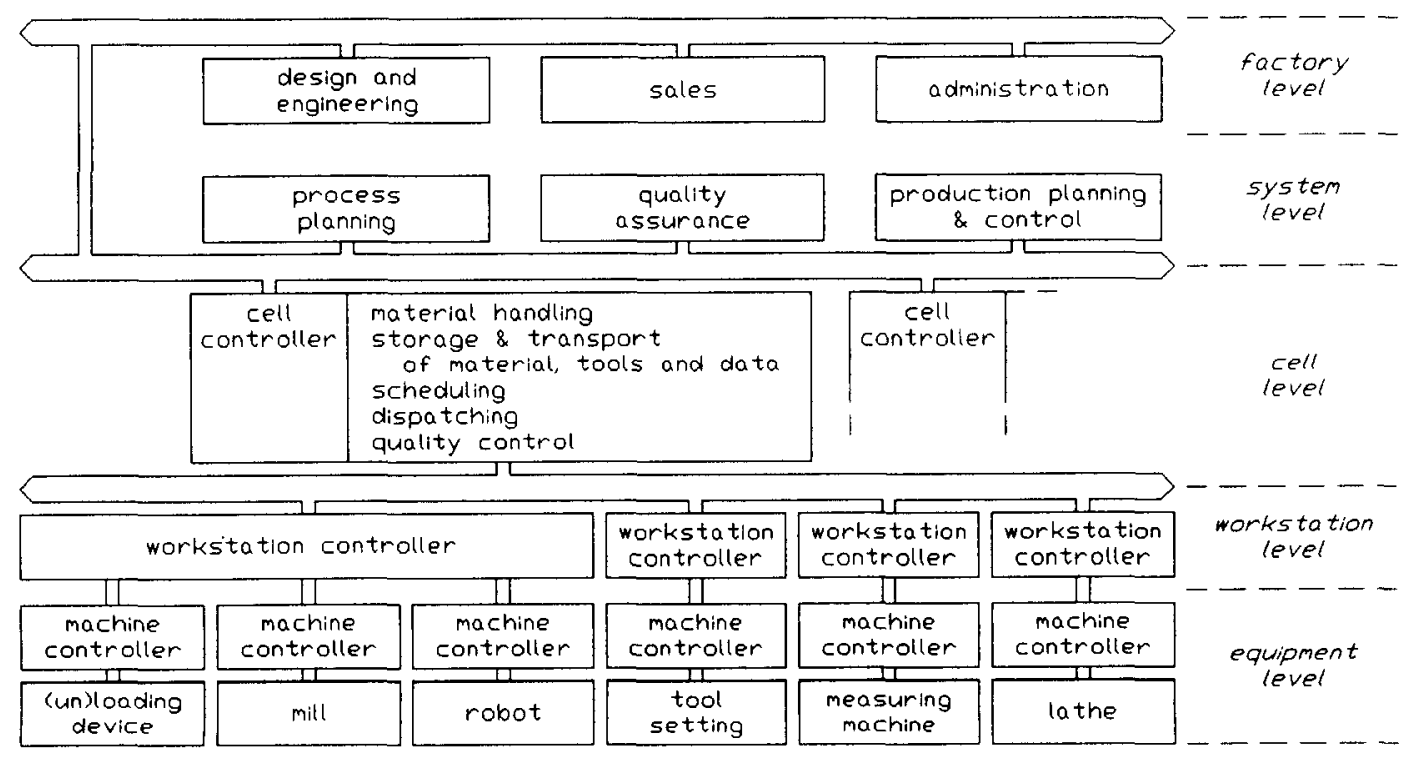

Fig. 1. CIM reference model. 
timely, efficient and cost-effective manner. Flexibility is defined as the capability of the system to react in real-time to the dynamic behaviour of the manufacturing environment. In the case of small batch part manufacturing, flexibility is also required to achieve effectiveness. Real-time, within the scope of PCS, means that the response times of the system must be within the values required by the different processes in the controlled environment. The response times in the PCS may vary from a second up to an hour; for example, events caused by the human operators may need a response time within the order of magnitude of one second, but in the case of re-scheduling under normal conditions (not caused by a disturbance) an hour could be enough. Critical events at equipment level like collisions, tool failure, etc. are not dealt with by the PCS, but must be handled by the lower level equipment control loops. In order to achieve a flexible, real-time control system, on-line connections are necessary with the equipment in the manufacturing environment.

Adaptability refers to the on-line connections of the PCS with the manufacturing environment and is defined as the capability of the system to deal with various types of manufacturing equipment from different vendors and different degrees of automation.

Human beings play, and will continue to play, an essential role in small batch part manufacturing. Therefore, an operator must be able to master and use the system, even when it has considerable inherent complexity. As the principal criterion to meet this requirement, the system must have a conceptual integrity and unity of view, which allows human beings to master the system as a whole.

The need for a low-cost system exist because small batch part manufacturing is usually carried out by small companies, who cannot afford expensive systems. The authors believe that these smaller companies, representing a significant part of the industry, can benefit most from the advantages of using the PCS.

\subsection{Functional description of the PCS}

The PCS consists of five, hierarchically structured modules shown in Fig. 2.6,8 The modules are scheduling, ${ }^{5}$ dispatching, workstation control, ${ }^{7}$ station control and monitoring and diagnostics. A brief description of the modules is given below.

The PCS receives capacity plans, consisting of production orders and the accessory process plans which have to be carried out in the coming time period, from system level (see CIM reference model). Based on the availability of the equipment and production hours, the production orders are scheduled into a workplan by the scheduling module. A workplan contains the sequence of jobs to be executed at the shop floor. A job represents either a process step of a process plan to be executed at a workstation or any of the auxiliary tasks, for example tool preparation, transport of materials, tools and information (NC part programs, tool offset data, etc.). For each job the start and the finish time is

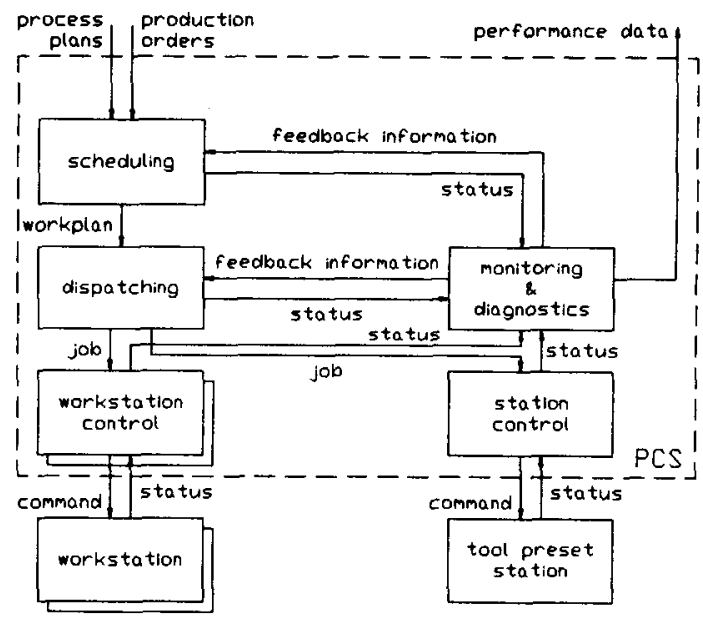

Fig. 2. Functional layout of the PCS.

calculated. This way of scheduling provides the advantage that at each moment it is known where a product should be and in what state it should be. This is important for the total control of the manufacturing cell.

The output of the scheduling module serves as a command for the dispatching module, which releases jobs to the different workstations, preset station(s) and stores within the cell, by using the information contained in the workplan and the status information, supplied by the monitoring and diagnostics module, about the progress of the jobs within the cell. When the dispatching module determines that a particular job cannot be started, because for example the required information or tools are not available yet, it is reported to the monitoring and diagnostics module. The output of the dispatching module serves as a command for workstation control and station control modules.

The monitoring and diagnostics (MD) module transforms the feedback information within the PCS. Status information is collected and transformed to the form required by the different modules and is passed on via the database of the PCS. The MD module generates user-configurable diagnostic reports and reports the performance of the cell to system level.

The workstation control (WC) module ${ }^{7}$ supplies a workstation with the required job information and coordinates the tasks of the equipment within a workstation. In addition, the WC module collects the status information within the workstation and passes it on to the MD module. The design of the WC module is based on the virtual workstation concept, i.e. the WC module operates within the PCS as a standard workstation offering the required functionality to execute a job autonomously. The cell level modules are not concerned with how the functions are performed; the WC module performs its functions by addressing both the equipment controllers and, when applicable, the operator of the workstation.

The station control (SC) module delivers the same functionality as the WC module but is dedicated to 
auxiliary manufacturing functions, e.g. tool presetting, fixturing and material storage. Within the scope of the development of the PCS, an SC module has been developed for a tool preset station. The SC module receives tool preset jobs from the dispatching module. A tool preset job represents the preparation of a complete tool set for a job which has to be performed at a workstation.

\subsection{Architecture of the PCS}

The hierarchically distributed control architecture (HDCA) of the PCS is shown in Fig. 3. Within the HDCA, a strict separation has been made between functions, information and communication. The modules are relatively independent, using information which is stored in the central database of the system. The modules are divided over two hierarchical levels, namely cell and workstation level. In addition, the modules of the PCS are geograpically distributed over the shop floor.

One computer is used for the general purpose modules at cell level and is called the cell computer. At the cell computer the scheduling, dispatching, and monitoring and diagnostics modules are allocated.

The other computers are used at workstation level for modules dedicated to the control of the shop floor equipment. The workstation control module is allocated at the so called machine interface unit computer (MIU). Each workstation has its own MIU which has an on-line connection with the equipment of a workstation. The station control module of the PCS is allocated at the so called preset interface unit computer (PIU). The tool preset station has an on-line connection to the PIU for sending the tool offset data of a tool set.

The cell computer used is an ICL System 25 running the UNIX operating system on a Motorola 68000 co-processor. The system has 12 Mbytes of main memory and 200 Mbytes of secondary storage. UNIX Interprocess Communications (IPC) facilities are used for easy intertask data transfer. The database package used is an off-the-shelf relational database management system from INFORMIX. This system uses SQL commands to access the data and an interface to ' $\mathrm{C}$ ' is available using Embedded $\mathrm{SQL} /{ }^{\mathrm{C}} \mathrm{C}$ ' statements. The SQL/'C' interface allows the modules to access the database easily.

The MIU, and also the PIU, is an IBM compatible $\mathrm{PC} / \mathrm{AT}$ running MSDOS 3.3 with graphic VDUs. The programming languages used are Modula 2, which offers a good development environment and pseudoconcurrency, and assembler for low level functions.

\section{A REAL-TIME CONTROL NETWORK FOR THE PCS}

This section describes the communication services provided by the control network and reviews the applied design requirements.

\subsection{Communication service requirements of the PCS}

A control network is needed for communication between the cell computer and the different MIUs and the PIU. The communication services supplied to the different modules, dispatching, monitoring and diagnostics, workstation control and station control, are derived from the functional requirements of the different modules.

Information exchange between the different modules of the PCS can be divided into two categories: messages, which include commands and status information, and files. The messages required are listed and described below.

1. Job control

2. Missing component

3. Status report

4. Stop and delay

5. Product quality

6. Operator note

7. Direct tool request

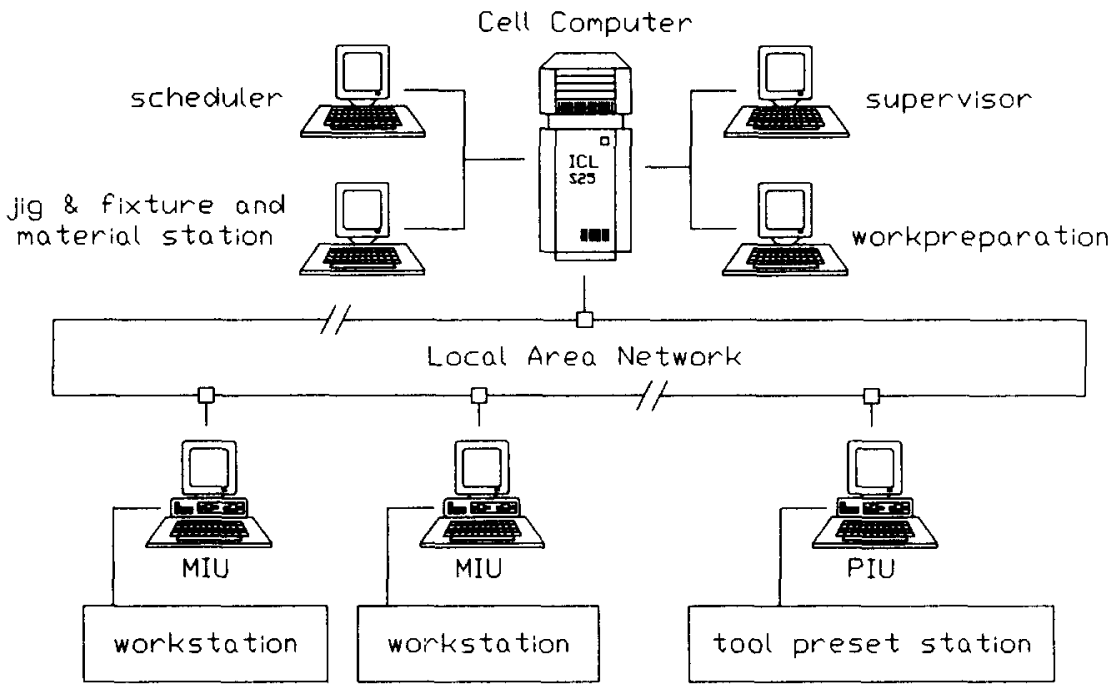

Fig. 3. Architecture of the PCS. 
The job control message is sent by the dispatching module to the workstation and station control modules to instruct the modules about the jobs which have to be executed in the coming period. In addition, the modules are given details about which materials, tools and information are required to perform each job. The job control message is also used to instruct the modules to cancel a particular job because of, for example, re-scheduling.

The missing component message is sent by the workstation control module or station control module to the dispatching module when a physical object (e.g. material or tools) or information (e.g. NC part program, tool set data) is missing as a consequence of which a job cannot be started.

The status report message reports the progress of the job at a workstation or a tool preset station. It is sent from the workstation control module to the monitoring and diagnostics module.

The stop and delay message is related to disturbances occurring at the shop floor and is sent by the workstation and station control modules. It informs the monitoring and diagnostics module about the reasons for a disturbance, like the breakdown of a machine tool, a material error, a program error, etc. The message also gives an estimate of the delay time. The estimate of the time the delay will last is given by the operator.

The product quality message is sent by a workstation control module to the monitoring and diagnostics module to give the number of products produced to date and the quality of each piece. In the case of rejected pieces, the reason for the reject is also given, e.g. machine failure, operator failure, etc.

The operator note message is sent on special occasions when the operator wants immediately to report serious problems to the cell supervisor, like delay expected or running out of blanks.

The direct tool request message is sent by a workstation control module to the tool preset station control module when a tool breakdown has occurred and no replacement tool is available at the workstation.

The file transfer between the different modules concerns information like NC part programs, operator instructions, tool offsets, job data, tool set data and product, jig and fixture drawings.

\subsection{Control network design requirements}

The most important design requirement with respect to communications between different computer systems is the ISO Open System Interconnection reference model. ${ }^{3}$ For communication within manufacturing the MAP Manufacturing Message Specification (MMS), version 3, and FTAM ${ }^{4}$ should be applied, or within the European context the CNMA standards apply. ${ }^{2}$ The applicability of MMS within the manufacturing environment dealt with by the PCS is still very limited. The existing manufacturing equipment is not capable of understanding MMS.
In addition, the future of MMS is not stable enough yet. MMS compatible equipment will probably become available but it is questionable whether it will be bought by the smaller companies, because of its applicability and cost reasons. Even if it is bought, it will still take a long time before the whole manufacturing environment can be controlled by using MMS, because equipment will be replaced only gradually.

The development of the PCS has been addressed to the improvement of production control in existing and developing small batch part manufacturing industry. Although the communication services required would perfectly fit into MMS and FTAM, it was decided not to follow the standards within the PCS for the reasons described above.

\section{DESCRIPTION OF THE DEVELOPED CONTROL NETWORK}

This section gives a description of the control network as it has been developed for the PCS based on the ISO/OSI reference model. The section is divided into two subsections. The first subsection describes the offthe-shelf products used and the second describes the applications which have been developed in order to integrate the control network into the PCS.

\subsection{Standard communications equipment}

The lower four layers of the ISO/OSI reference model have been accomplished by using the ICL Open System Local Area Network (OSLAN) which is based on the following ISO standards:

$\begin{array}{ll}\text { Transport layer: } & \text { ISO } 8073 \text {, Class } 4 \\ \text { Network layer: } & \text { ISO } 8473 \\ \text { Data link layer: } & \text { ISO } 8802 / 2 \text { (IEEE } 802.2 \text { ) } \\ \text { Physical layer: } & \text { ISO } 8802 / 3 \text { (IEEE } 802.3 \text { ). }\end{array}$

Thin wire Ethernet was chosen because it is the most popular standard adopted and it was possible to buy circuit boards and firmware for the computers used at competitive prices. The hardware and software available is well-proven and reliable. It can handle the electrical noise generated by the $\mathrm{NC}$ machinery, etc. and is physically robust enough to be routed around the different areas where connections are needed.

OSLAN provides a standard interface to the transport layer for file transfer, terminal facilities and communication between different application processes. Two application processes communicate with each other in a logical fashion by using the procedural interfaces provided by the Application Data Interchange (ADI) interface. The ADI interface consists of four basic functions, OpenADI, ReadADI, WriteADI and CloseADI, and provides the different application processes with a guaranteed end-to-end data exchange.

\subsection{The application interface}

The design of the "top layers" of the control network has also been developed according to the ISO/OSI 
reference model. The session, presentation and application layers are described separately in the following paragraphs.

The session layer is where an end-to-end connection is actually established. The design of the session layer is based on the responder/initiator mechanism. One application opens a connection and waits for a successful completion of the connection. The other end tries to make its own connection by making a call at regular time intervals until a connection is established.

The presentation layer has a minimal impact in this instance because the data on both systems are held in the same format, namely ASCII, and the only differences are due to the operating systems, namely UNIX and MSDOS.

The application layer provides the actual communication services, as described in Subsection 2.1, to the different modules at the cell computer, MIUs and PIU. The layer consists of two interfaces. The first interface is called the command/status interface which provides message transfer services. It offers a full duplex link to allow either end to initiate a transfer and operates without any built-in delays to provide a very fast (real-time) message exchange facility. The command/status interface also takes care of data integrity, guaranteed application process to application process transfer, error recovery, recovery from a broken LAN or an application restart, and a startup/ closedown of the whole system. In the latter case it is also possible to reconfigure the system.

The second interface, called the file transfer interface, provides file transfer services. Files can be sent or received by either module. The file transfer handles only one file at a time and completes that transfer before initiating another.

The dispatching and monitoring and diagnostics modules know the identities of the different workstation control modules but do not know the physical location or the physical connections for the machine tools to which they interface. The mapping of the WC modules to the network is recorded in the database to allow the control network to route the messages correctly.

Each workstation control and station control module has its own full duplex link for message transfer and its own file transfer link. All the tasks related to the control network, running on the different computers, remain dormant until either a message from another task or a data packet from the network is received. The "dormant mechanism" is handled either by the UNIX kernel and hardware on the cell computer or the pseudo-currency kernel developed and hardware on the MIU/PIU.

\subsection{Security of messages}

The PCS relies on data integrity of the control network to maintain a coherent and consistent system. Although the OSLAN provides data integrity, application to application communication is still not guaranteed. Therefore, an application to application communication security mechanism has been developed, called the sliding window (SW) mechanism.

Briefly, the SW mechanism saves all of the transmitted messages on hard disc until they are acknowledged by the application at the receiving end. Every message transmitted is given a unique identifier which increases by one each time. The receiver of a message knows the identifier of the last message received and stored. It sends the identifier with any message it transmits. Then the transmitter knows that all messages sent with an identifier number less than or equal to this number have been received successfully and it can delete them from the hard disc. If there is an error then the messages can be recovered and resent.

\section{RESULTS}

A prototype of the PCS has been installed and tested in a (pilot) plant, called Morskate Aandrijvingen B.V., located in Hengelo, The Netherlands. The PCS has been in use now for 9 months and the continuous operation of the control network has proven to be very reliable. The PCS has 10 MIUs connected on-line to CNC controlled machine tools. The PIU is connected on-line to a tool preset station.

The thin wire Ethernet hardware used has proven to be very reliable. No problems have been encountered related to electrical noise generated by the $\mathrm{CNC}$ machine tools. A typical snapshot of the network shows that with over 300,000 layer 4 transmissions there were only 600 layer 4 re-transmissions.

The SW mechanism of the control network guarantees the data transfer between the different application processes. The error recovery mechanism has been tested thoroughly by simulation of all possible disturbances, by for example disconnecting cables, and has proven to work perfectly. The performance is more than adequate and it has been demonstrated that, in the worst case, with 10 MIUs each sending three messages, at the same time, to the cell computer the last message causes the database to be updated within $10 \mathrm{~s}$. The concept of the PCS development allows a manufacturing cell to contain up to 20 or 30 workstations. Given the performance of the control network for $10 \mathrm{MIUs}$ and one PIU, the real-time requirement of the PCS can be met, even with a maximum of workstations connected on-line.

\section{CONCLUSIONS}

In this paper, the development of a control network has been described which forms an integral part of a shop floor control system for small batch part manufacturing. The control network design has been based on international standards according to the ISO Open System Interconnection reference model and the components used are available as stock items at very competitive prices. Although the goal has been to follow the CNMA Manufacturing Message Specification and FTAM, it is unfortunately not possible yet to use it. The main reason for this is that MMS-compatible equipment is not yet available and that it will 
probably take a long time before it can be made available to and accepted by companies dealing with small scale production. These companies represent a great part of the European industry and require inexpensive solutions.

Nowadays, these companies do not have adequate tools for flexible and efficient control of shop floor activities at their disposal. The PCS concept has proven to provide a fair solution for this. The control network developed has proven that it can supply a manufacturing environment, consisting of equipment from different vendors with different levels of automation, with a reliable, low cost, real-time communication facility.

\section{REFERENCES}

1. Biemans, F. P. M.: Manufacturing Planning and Control: a Reference Model, Amsterdam, Elsevier, 1990. Series: Manufacturing Research and Technology; part 10.

2. CNMA ESPRIT 955: CNMA implementation guide, Revision 3.1. Dec. 1987.
3. ISO TC974/SC16: Information processing systems, open system interconnection basic reference model. International standards, technical report, 1983.

4. MAP: Manufacturing Message Specification, draft 6 . EIA IE-31/ISO 2nd DP 9506/RS511, 1987.

5. De Swaan Arons, H., Riewe, D.: Integration of dynamic expert scheduling in production control. Proceedings of the ESPRIT Conference 1989, Proceedings of the 6th Annual ESPRIT Conference, Brussels, 1989.

6. Tiemersma, J. J.: The development of a production control system for small batch part manufacturing by the ESPRIT project 809. Proceedings of the ESPRIT Conference 1988, Proceedings of the 5th Annual ESPRIT Conference, Brussels, 1988.

7. Tiemersma, J. J., Kals, H. J. J.: The design of a machine interface unit for real-time control within flexible manufacturing systems. Proceedings of the 4th International Conference on the Manufacturing Science and technology of the Future '89, Stockholm, 1989.

8. Tiemersma, J. J., Kals, H. J. J.: A real-time monitoring and control system for small batch part manufacturing. Proceedings of the Manufacturing International '90 Conference, Atlanta, U.S.A., 1990.

9. Yeomans, R. W., Choudry, A., Hagen, P. J. W. ten: Design Rules for CIM System. Amsterdam, Elsevier, 1985. 